\title{
El cuerpo en la filosofía de Merleau-Ponty
}

\author{
The body in the Merleau-Ponty's Philosophy
}

ANTONINO FIRENZE*

\begin{abstract}
Resumen: El propósito de esta contribución es exponer los elementos esenciales de la filosofía del cuerpo de Merleau-Ponty. En un primer momento describiré la función trascendental del cuerpo como cogito tácito planteada en Phénoménologie de la perception, para a continuación poner de relieve la profundización ontológica que, con respecto a ésta, se esboza en Le visible et l'invisible. En efecto, en este contexto tardío, la comprensión de la corporeidad (lo visible) depende constitutivamente de su vínculo con el pensamiento (lo invisible). Mostraré que este quiasmo de visible e invisible remite de modo original al principio de productividad inmanente a la carne del mundo en cuanto que dimensión ontológica fundamental.
\end{abstract}

Palabras clave: cuerpo, Merleau-Ponty, ontología, cogito tácito, quiasmo, carne del mundo

\begin{abstract}
The aim of this paper is to present the essentials elements of Merleau-Ponty's philosophy of body. I will describe the transcendental function of the body as a tacit cogito outlined by Phénoménologie de la perception, in order to highlight, in contrast with this, the ontological deepening roughed out by Le visible et l'invisible. Indeed, in this late context, the understanding of the corporeality (the Visible) depends in a constitutive way on his link with the thinking (the Invisible). I will show that this Visible-Invisible Chiasm adheres in an original way to the productivity principle immanent to the flesh of the world as a fundamental ontological dimension.
\end{abstract}

Key words: body, Merleau-Ponty, ontology, tacit cogito, Chiasm, flesh of the world.

\section{El cuerpo como trascendental}

Desde la publicación de sus primeras dos obras hasta el escrito póstumo Le visible et l'invisible, el problema teórico fundamental que preocupó a Merleau-Ponty fue el de superar el clásico dualismo metafísico de cuerpo y conciencia, paradigmáticamente representado en

Fecha de recepción: 10/06/2016. Fecha de aceptación: 20/07/2016.

* Antonino Firenze es profesor agregado de Filosofía Contemporánea en el Departamento de Humanidades de la Universitat Pompeu Fabra de Barcelona. Sus principales líneas de investigación están relacionadas con la fenomenología y la antropología filosófica. Entre sus publicaciones destacan Il corpo e l'impensato. Saggio su Merleau-Ponty, Milano, Mimesis, 2011, e «Intercorporéité et interanimalité. La philosophie de la nature de Merleau-Ponty et l'esquisse d'une nouvelle ontologie de la vie», en K. Novotný, P. Rodrigo, J. Slatman, S. Stoller (Ed.), Corporeity and Affectivity. Dedicated to Maurice Merleau-Ponty, Leiden-Boston, Brill, 2014, pp. 139-148. Correo electrónico: antonino.firenze@upf.edu 
la modernidad por la filosofía cartesiana․ Según el planteamiento crítico que recorre toda la obra del pensador francés, en efecto, el dualismo cartesiano sería el responsable de haber enfocado la relación cuerpo-conciencia desde una perspectiva profundamente dicotómica en la que se enfrentarían una forma de ser en cuanto conciencia con otra en cuanto cuerpo-cosa. Dicho con otras palabras, por un lado, una conciencia absolutamente libre, que constituye autónomamente la realidad del mundo mediante las cogitationes ciertas y evidentes del cogito. Y, por el otro, un cuerpo exterior a la conciencia, concebido por tanto de la misma manera que los objetos físicos estudiados por las ciencias naturales. Esta dicotomía entre un punto de vista reflexivo-idealista y uno causalista-realista desemboca, según MerleauPonty, en una "diplopía" en cuyo interior el hombre se ve desdoblado. ${ }^{2}$ Es precisamente esta diplopía ontológica que Merleau-Ponty se esforzó de repensar a lo largo de su obra para conducir la interrogación filosófica más allá del impasse dualista.

Dicho esto, cabría señalar que sobre todo a partir de Phénoménologie de la perception, y en la estela de la fenomenología husserliana, el filósofo emprende su crítica del dualismo a partir de una rehabilitación fenomenológica de la corporeidad subjetiva, o, tal y como suele escribir allí Merleau-Ponty, de la subjetividad entendida como "cuerpo propio" (corps propre). Como es generalmente admitido por la crítica, con este término el filósofo suele referirse a la noción husserliana de Leib (cuerpo vivo), en cuanto cuerpo sujeto, «Yo puedo» y unidad originaria e implícita de la conciencia, contrapuesta a la de Körper (cuerpo físico), en cuanto cuerpo objetivado por las ciencias naturales.

Por lo tanto, la descripción fenomenológica de la diferencia ontológica entre Körper y Leib es un elemento teórico esencial con el que medirse a la hora de devolver la debida centralidad a la corporeidad subjetiva frente a las pretensiones cognoscitivas de la corporeidad objetiva exhibidas por el Cogito cartesiano. En suma, si la experiencia perceptiva del cuerpo propio se opone de modo radical al movimiento dicotómico de separación de sujeto y objeto, se tratará de rehabilitarlo diferenciándolo claramente de la representación científica del cuerpo objetivo como partes extra partes y fragmento de la res extensa. Como el propio Merleau-Ponty afirma: «Me comprometo con mi cuerpo entre las cosas, estas coexisten conmigo como sujeto encarnado, y esta vida dentro de las cosas nada tiene en común con la construcción de los objetos científicos», ya que «es por mi cuerpo que comprendo al otro, como es por mi cuerpo que percibo "cosas" $»^{3}$. Lo que se quiere aquí poner de relieve es que solo a partir del cuerpo propio, es decir de una subjetividad encarnada en un cuerpo vivo, resulta posible acceder al mundo y a los otros cuerpos vivos, y esto de forma preliminar a la intervención de la conciencia reflexiva volcada a pensar el mundo según los parámetros de la verdad objetiva construida por la ciencia. Por lo tanto, es en este "saber singular" que

1 Cfr. La structure du comportement, Puf, Paris 2002 (1942), trad. cast. de E. Alonso, La Estructura del comportamiento, Hachette, Buenos Aires, 1957; Phénoménologie de la perception, Paris, Gallimard, 2003 (1945), trad. cast. de J. Cabanes, Fenomenología de la percepción, México D.F. - Buenos Aires, Planeta De Agostini, 1993; Le visible et l'invisible, seguido de: Notas de trabajo, texto establecido por C. Lefort, Gallimard, París, 2003 (1964), trad. cast. de E. Consigli, B. Capdevielle, Lo visible y lo invisible, Buenos Aires, Nueva Visión, 2010.

2 Sobre esta noción de "diplopía", a la que acompaña a menudo como sinónimo la de "estrabismo", cfr. Lo visible y lo invisible, op. cit., pp. 149-150 nota (nota de trabajo enero de 1959). Sobre el trasfondo blondeliano de esta noción véase Saint Aubert, E. de, Vers une ontologie indirecte. Sources et enjeux critiques de l'appel à l'ontologie chez Merleau-Ponty, Paris, Vrin, 2006, pp. 123-130.

3 Fenomenología de la percepción, op. cit., pp. 202-203. 
tenemos del mundo y de los demás hombres, sólo porque "somos un cuerpo", que la ciencia y la filosofía se descubren enraizadas antes que en ningún conocimiento tético, discursivo y puramente intelectual.

Coherentemente con este planteamiento teórico, la Phénoménologie de la perception defiende esta primacía de la corporeidad perceptiva sobre la objetiva recurriendo a menudo al término "cogito tácito". Desde un punto de vista pre-reflexivo, este indicaría el cuerpo propio como una forma de autoconciencia primordial, anterior a la expresión verbal y al concepto. En este contexto por lo tanto el término de cogito tácito viene a designar el lugar de fundación del cogito reflexivo, hallando por ello en el cuerpo propio la condición de posibilidad de la experiencia y el centro de irradiación de un contacto "directo" del sujeto perceptivo con el mundo. La noción de cogito tácito acabaría de este modo coincidiendo con el núcleo "trascendental" de la subjetividad, a partir del cual refundar el sentido producido por la percepción en contraste con la abstracción intelectualista de una primacía metafísica del pensamiento reflexivo sobre la existencia corpórea.

Como se puede leer en el siguiente fragmento, en el que toma como blanco de su crítica la segunda meditación metafísica, ${ }^{4}$ Merleau-Ponty desmonta la pretensión de Descartes de someter la evidencia indudable de su existencia al ser pensante, identificando la noción de cogito tácito con una forma de autoconciencia originaria y, por ello, más auténtica: «ni siquiera podría leer el texto de Descartes, si no estuviera, anteriormente a toda palabra, en contacto con mi propia vida y mi propio pensamiento y si el Cogito hablado no hallara en mi un Cogito tácito. [...] Todo el problema radica en comprender bien el Cogito tácito, en no poner en él más de lo que verdaderamente comprende, y en no hacer del lenguaje un producto de la conciencia con el pretexto de que la conciencia no es un producto del lenguaje. Ni la palabra, ni el sentido de la palabra son, en efecto, constituidos por la conciencia». ${ }^{5}$ Para Merleau-Ponty, por lo tanto, el origen del pensamiento se hallaría, en efecto, en la palabra, pero entendida ésta como gesto expresivo del cuerpo y ya no como el resultado del poder constituyente de la conciencia reflexiva. En este sentido si afirma que el «Cogito tácito, la presencia de sí a sí, al ser la existencia misma, es anterior a toda filosofía», ${ }^{6}$ lo hace para recuperar la génesis opaca del pensamiento, es decir el momento en el que la palabra, en cuanto gesto del cuerpo, es atravesada por el silencio a la vez que se esfuerza por decir su vínculo originario con el mundo. En otros términos, se trata de hallar por debajo de la "palabra hablada" el silencio primordial del cuerpo justo en el momento en que la "palabra hablante" rompe este silencio con su gesto expresivo7.

4 Cfr. Descartes, R., Meditaciones metafísicas, Madrid, Alfaguara, 1977, pp. 23-30.

5 Fenomenología de la percepción, op. cit., pp. 411 (trad. modificada).

6 Cfr. Ibid., p. 413; véase también: Ibid., p. 397: « Nuestro cuerpo en cuanto se mueve, eso es, en cuanto es inseparable de una visión del mundo y es esta misma visión realizada, es la condición de posibilidad [...] de todas las adquisiciones que constituyen el mundo cultural».

7 Sobre la diferencia entre "palabra hablante" (parole parlante) y "palabra hablada" (parole parlée) véase el capítulo titulado «El cuerpo como expresión y la palabra» (cfr. Ibid., pp. 191-216). En este contexto, de hecho, Merleau-Ponty trata de mostrar que el sentido operante en la parole parlante, antecediendo la relación entre significante-sujeto y significado-objeto, es pre-comprendido por el cuerpo como significado afectivo procedente del cuerpo de las cosas y del cuerpo de los otros sujetos. Por debajo del significado lógico-categorial de las palabras aparece por lo tanto un significado gestual mediante el cual el sujeto recoge de modo originario el esfuerzo de su cuerpo para ser-en-el-mundo. Sobre este punto véase Dastur, F., Le corps de la 
Sin embargo, este planteamiento deja sin resolver un aspecto fundamental de la cuestión. La primacía atribuida al cuerpo propio en la genealogía de la conciencia llevada a cabo en la obra de 1945 no permite enfocar de modo adecuado el problema del pasaje del sentido perceptivo al sentido lingüístico. Para decirlo de otro modo, a causa de la identificación fenomenológica del Leib con la vida silenciosa y anónima del cogito tácito, Merleau-Ponty acabaría desatendiendo la especificidad ontológica del lenguaje. Pero eso no es todo. Más radicalmente, al concebir el cogito tácito como el lugar de fundación del cogito reflexivo, es decir como centro de la experiencia de la conciencia, la Phénoménologie de la perception terminaría haciendo del cuerpo propio un nuevo trascendental, recayendo así en la actitud subjetivista propia de la metafísica. ${ }^{8}$

\section{El quiasmo de visible e invisible}

No obstante, como se puede apreciar en los textos posteriores a Phénoménologie de la perception, este punto de vista se revela insuficiente a la mirada del propio Merleau-Ponty. En otro lugar, ${ }^{9}$ he mostrado que en los textos de los años 50 no se expresaría tanto un rechazo del anterior planteamiento sino más bien una radicalización de la filosofía del cuerpo en dirección de lo que el filósofo considera el suelo ontológico fundamental de este cuerpo, a saber: el Ser en cuanto carne del mundo. Es esta radicalización en sentido ontológico de su temprano enfoque fenomenológico sobre el cuerpo propio lo que llevará a Merleau-Ponty a concebir el Ser del mundo como el lugar en el que se realiza la metamorfosis del cuerpo en palabra y de la palabra en cuerpo. Lo que de este modo se acaba mostrando es la "pregnancia" del sentido ideal y del sentido mudo del cuerpo por cuanto ambos proceden de esta dimensión ontológica fundamental.

Gracias a esta nueva perspectiva, el propio Merleau-Ponty, en las notas de trabajo de Le visible et l'invisible, retoma de modo autocrítico la noción de cogito tácito defendida en Phénoménologie de la perception. Tomemos como ejemplo este pasaje: «El Cogito de Descartes (la reflexión) es operación sobre reflexiones, enunciado de relaciones entre ellas (y las propias significaciones sedimentadas en actos de expresión). Presupone pues un contacto prerreflexivo de uno consigo mismo [...] o un cogito tácito (ser cerca de sí mismo) - así lo he razonado en $P h . P$. ¿Es correcto? Lo que llamo el cogito tácito es imposible. Para tener la idea de «pensar» (en el sentido del «pensamiento de ver y de sentir»), [...] es necesario tener las palabras». ${ }^{10}$

parole, en Id., Chair et langage, La Versanne, Encre Marine, 2001, pp. 49-68, en part. p. 60; más en general: Thierry, Y., Du corps parlant. Le langage chez Merleau-Ponty, Bruxelles, Ousia, 1987.

8 Sobre todo Nancy ha detectado en este enfoque fenomenológico de la corporeidad la pervivencia de cierto consciencialismo metafísico: concebir el cuerpo como "interioridad primera", "presencia absoluta" y "organon del sentido", implicaría pensarlo todavía en la estela de la metafísica de la presencia como evidencia autorepresentada del fundamento. Véase: Nancy, J.-L., Corpus, Paris, Éditions Métailié, 2000.

9 Il corpo e l'impensato. Saggio su Merleau-Ponty, Milán, Mimesis, 2011, pp. 145-179, en donde se ha puesto especialmente el acento en la reciprocidad metodológica de fenomenología y lingüística (Saussure, Jakobson), sobre cuya base Merleau-Ponty ha concebido el entrelazamiento ontológico de la diacriticidad del signo lingüístico con la opacidad estructural de la experiencia corpóreo-perceptiva.

10 Lo visible y lo invisible, op. cit., p. 154 (enero 1959). Sobre la autocrítica relativa al concepto de Cogito tácito véanse: Taminiaux, J., L'experience, l'expression et la forme dans l'itineraire de Merleau-Ponty, en Id., Le 
Criticando de este modo por ingenua la idea del cogito tácito como fundamento de la experiencia subjetiva, el autor lamenta especialmente haber creído que era posible adecuar descriptivamente la conciencia reflexiva a este fondo pre-verbal, cuando en realidad no había hecho otra cosa que describir el silencio mediante un discurso basado íntegramente sobre las "virtudes del lenguaje". De este modo lo que se cuestiona es el cuerpo propio como coincidencia psicológica y núcleo profundo de la subjetividad, el cuerpo propio como referente originario y "trascendental". Leamos otro pasaje significativo a este respecto: «El Cogito tácito, por supuesto, no resuelve esos problemas. Revelándolo como lo hice en $P h$. P. no he arribado a una solución [...]: por el contrario, he planteado un problema. El Cogito tácito debe hacer comprender cómo el lenguaje no es imposible, pero no puede hacer comprender cómo es posible - Queda pendiente el problema del pasaje del sentido perceptivo al sentido referido al lenguaje, [...] el lenguaje realiza quebrando el silencio lo que el silencio quería y no obtenía. [...] Se trata de aprehender lo que, a través de la comunidad sucesiva y simultánea de los sujetos hablantes, quiere, habla, y finalmente piensa». ${ }^{11}$ Lo que está aquí en juego es por tanto la profundización de la interrogación fenomenológica del sujeto corpóreo hacia la interrogación ontológica del sentido de ser del suelo (Boden) originario en cuyo sí se manifiesta el sentido de ser específico del sujeto. Es decir, el sentido de ser de un Ser que "quiere, habla y piensa" en y a través del sujeto corpóreo-hablante.

No obstante, no se trata simplemente de indicar el límite teórico de su enfoque anterior sino más radicalmente de poner de relieve una paradoja ineludible y constitutiva del discurso filosófico en cuanto tal. Dicho de otro modo, si bien se hace necesario lograr pensar que el cuerpo viviente es tal porque está íntimamente ligado al lenguaje, por lo que hay que evitar hipostasiar el cuerpo y su cogito, también es cierto que revelar la incrustación del lenguaje en la corporeidad no deja de tener consecuencias decisivas en relación con la pretensión de auto-referencialidad y transparencia representativa que, tradicionalmente, han contribuido a fundamentar la voluntad constituyente del discurso filosófico. El problema central de la reflexión de Merleau-Ponty es ahora comprender de qué modo el ser corpóreo visible se diferencia de sí mismo hasta convertirse en ser lingüístico invisible. Más precisamente, lo que el filósofo pretende esbozar es esa característica impensada del Ser en virtud de la cual la palabra emergente del cuerpo se manifestaría ante todo como algo que no le pertenece al cuerpo propio, en calidad de su específica prerrogativa trascendental y antropológica, siendo más bien fruto de una expresión operante de modo preliminar en el Ser. ${ }^{12}$

Como se verá a continuación, es justamente la noción de quiasmo de cuerpo y palabra, de percepción y lenguaje, o para decirlo con el título de la obra, de lo visible y lo invisible, lo que permite a Merleau-Ponty repensar de manera radical el dualismo metafísico y formular así su "nueva ontología". Al respecto, en otra nota de trabajo, se puede leer que «hay un

regard et l'excédent, La Haye, Martinus Nijhoff, 1977, pp. 90-115; Barbaras, R., De la phénoménologie du corps à l'ontologie de la chair, en Id., Le tournant de l'experience. Recherches sur la philosophie de MerleauPonty, Paris, Vrin, 1998, pp. 95-136; Thierry, Y., Le «cogito» comme expérience sensible, en Barbaras, R., Robert, F. (ed.), Notes de Cours sur L'origine de la géométrie de Husserl. Suivi de «Recherche sur la phénoménologie de Merleau-Ponty», Paris, PUF, 1998, pp. 255-268.

11 Lo visible y lo invisible, op. cit., p. 158.

12 Como señala generalmente la crítica, la ontología defendida en este tardo contexto parecería deudora del pensamiento heideggeriano posterior a la Kehre. Este tema lo he tratado en Firenze, A., «I segni di una nuova concezione dell'essere. Merleau-Ponty lettore di Heidegger», Chiasmi international, no 9, 2007, pp. 169-190. 
cuerpo del espíritu y un espíritu del cuerpo» solo porque hay «un quiasmo entre ellos»; y el espíritu se manifiesta como «el otro lado del cuerpo» ya que no es posible pensar el ser como un espíritu que no esté «acompañado por un cuerpo» y que no se establezca «en este suelo». ${ }^{13}$ Como he tenido ocasión de señalar en otro lugar, este "suelo" es el mundo como Boden de todo lo posible, es decir la Tierra de la que habla Husserl en el manuscrito de 1934 Umsturz der Köpernikanischen Lehre. ${ }^{14}$ Reconduciendo su reflexión a este texto, MerleauPonty se da cuenta de que con respecto a la Tierra, a su cuerpo-suelo (Boden-Körper), el cuerpo del sujeto ya no puede pensarse como cuerpo propio, porque el movimiento de las cosas y de los "otro cuerpos vivos" atraviesan al sujeto, entendido como cuerpo que percibe de modo originario la trascendencia de la corporeidad del mundo como una extrañeza y una alteridad que son internas a sí mismo. En su contacto primordial con la Tierra, el cuerpo vivo del sujeto se revela como sujeto-objeto (Leib-Körper) simultáneamente, ya que como individualidad que procede de la generalidad corpórea de la Tierra está entrelazado al cuerpo del mundo como su tejido ontológico fundamental. Es justamente mediante la noción de quiasmo que se puede desarticular toda distinción rígida de interior y exterior, de propio y ajeno, ya que la experiencia que procede de la generalidad corpórea de la Tierra es simultáneamente experiencia de pasividad y actividad, de una posesión marcada por un desposeimiento.

Como escribe el filósofo en otra nota de trabajo: «la idea del quiasmo, es decir: toda relación con el ser es simultáneamente aprehender y ser aprehendido, la aprehensión es aprehendida, está inscrita y se inscribe en el mismo ser que ella aprehende. A partir de eso, elaborar una idea de la filosofía: ella no puede ser aprehensión total y activa, posesión intelectual, puesto que lo que hay para aprehender es un desposeimiento - Ella no está sobre la vida, sobrevolándola. Está debajo de ella. Es la experiencia simultánea de lo que aprehende y de lo aprehendido en todos los órdenes». ${ }^{15}$ Irreductible tanto a una oposición de interior y exterior como a una secreta coincidencia de los términos o a una fusión intuitiva de los opuestos, el quiasmo se revela como el lugar de un cruce entre sujeto y mundo, interioridad y exterioridad, actividad y pasividad; o sea, el lugar en el que se abre el horizonte intotalizable del ser. Como se puede leer en otra nota de trabajo: «Quiasmo yo-el mundo, yo-otro, quiasmo mi cuerpo-las cosas, realizado por el desdoblamiento de mi cuerpo en adentro y afuera - y por el desdoblamiento de las cosas (su adentro y su afuera). Es posible porque hay esos 2 desdoblamientos: la inserción del mundo entre las 2 hojas de mi cuerpo, la inserción de mi cuerpo entre las 2 hojas de cada cosa y del mundo. Esto no es antropologismo: al estudiar las 2 hojas debemos encontrar estructura del ser - Partir de esto: no hay identidad, ni no-identidad o no-coincidencia, hay adentro y afuera girando uno en torno al otro». ${ }^{16}$

El quiasmo, por tanto, está pensado como una dehiscencia de lo visible exterior en lo invisible interior y viceversa; es decir, como una relación abierta e irreductible a la unidad

13 Lo visible y lo invisible, op. cit., p. 228 (junio de 1969).

14 Cfr. Husserl, E., «Umsturz der Köpernikanischen Lehre in der gewöhnlichen weltanschaulichen Interpretation», en Farber, M. (ed.), Philosophical Essays in Memory of E. Husserl, Cambridge, Harvard University Press, 1940, pp. 307-325. Sobre este punto me permito remitir a Firenze, A., «Mundo-de-la-vida, intercorporeidad, Tierra: Merleau-Ponty y la fenomenología», Phainomenon, n 20, 2010, pp. 129-142.

15 Lo visible y lo invisible, op. cit., p. 234 (noviembre de 1960).

16 Ibid., p. 232 (noviembre de 1960). 
simple de la identidad del Ser consigo mismo. Lo visible y lo invisible en el sujeto están envueltos por una torsión propia de la estructura abierta de la cosa y del mundo. El quiasmo de externo e interno, de cuerpo y pensamiento, como el de empírico y trascendental, manifiesta, entonces, un movimiento a través del cual el ser del mundo, y no la conciencia subjetiva, reproduce su inagotable trascendencia inmanente. ${ }^{17}$ Desde este punto de vista, la palabra operante en el pensamiento resulta ser co-extensiva al simbolismo mudo del cuerpo justamente porque «prolonga en lo invisible, extiende a las operaciones semánticas la pertenencia del cuerpo al ser y la pertinencia corpórea de todo ser, que me es definitivamente comprobada por lo visible». ${ }^{18}$ Es esta co-pertenencia que la filosofía, en cuanto reactivación indirecta de la palabra operante en el lenguaje, debe poner en el centro de su reflexión con vistas a «reconocer una idealidad que no es ajena a la carne, que le da sus ejes, su profundidad, sus dimensiones». ${ }^{19}$

La participación lateral e indirecta en esa productividad simbólica intrínseca al Ser corpóreo-sensible abre permanentemente el campo de lo decible transformando las propias estructuras de lo visible mudo en lo invisible hablante-pensante. Al lenguaje mudo de la percepción y del cuerpo se debe acompañar el lenguaje hablante del pensamiento en cuanto "sublimación", por parte del ser humano, de un poder de expresión ya operante en el cuerpo del mundo. La estructura muda de lo visible es tal que en él todas las posibilidades del lenguaje están presentes de modo implícito pero siempre a punto de expresarse. Merleau-Ponty pretende aquí afirmar el carácter profundamente fecundo y productivo del Ser en cuanto "vientre de los posibles"; es decir de un Ser que envuelve lo humano y hace de éste el lugar de su propia expresión, que nace con la percepción muda del cuerpo y se hace mediante la negatividad de la palabra operante en el lenguaje.

En suma, lo que se opone ahora al cogito tácito, concebido en su momento como ambigua coincidencia psicológica del cuerpo consigo mismo y con el mundo, es el desajuste (écart) del cogito corpóreo consigo mismo y su desposeimiento por parte de un Ser que lo determina y constituye como carne en el y del mundo. La expresión que surge de la carne del mundo a través del cuerpo es posible gracias a este desajuste consigo mismo que no es el yo corpóreo a determinar, ya que es fruto de una "negatividad natural" que está siempre ya dada de forma preliminar. Como se indica en otro pasaje, se trata de comprender la inherencia de lo invisible lingüístico con respecto a lo visible corpóreo en los términos de una potencia o trama latente que forra desde atrás la evidencia carnal del mundo para dejarse captar por el pensamiento de modo indirecto y negativo: «Nuestra tesis es que hace falta ese HAY [IL Y $A]$ de inherencia, y nuestro problema es mostrar que pensamiento, en el sentido restrictivo (significación pura, pensamiento de ver y sentir) sólo se comprende como cumplimiento por otros medios del deseo del HAY, por sublimación del HAY y realización de un invisible que es exactamente el reverso de lo visible, la potencia de lo visible». ${ }^{20}$

17 No comparto la crítica según la cual Merleau-Ponty, no habiendo superado el dualismo de empírico y transcendental, habría quedado ligado a una forma refinada de filosofía de la consciencia. Cfr. Barbaras, R., «Le problème du chiasme», en Studia Phanomenologica, Vol. III, n 3-4, 2003, pp. 15-20.

18 Lo visible y lo invisible, op. cit., p. 109.

19 Ibid., p. 137.

20 Ibid., p. 131 nota (trad. modificada). 
Con toda evidencia, lo invisible al que se refiere aquí el filósofo no es un invisible absoluto, lugar metafísico de la intuición originaria o de la adecuación del pensamiento al ser; más bien, lo invisible se entiende como esa negatividad o ausencia de la que surge el mundo visible de los cuerpos vivos y de los cuerpos de las cosas. Lo invisible es lo que se da como ausencia en la inminencia perceptiva y que, gracias a esta negatividad, genera en el sujeto corpóreo-sensible la cavidad en cuyo interior el mundo visible puede devenir decible y pensable. Lo invisible «está allí sin ser objeto, es la trascendencia pura», ya que todos los visibles están enraizados sobre un núcleo de ausencia o negatividad que a la vez funge de apertura para que lo posible llegue a existir en lo visible. ${ }^{21}$ Lo visible, por ende, se presenta como horizonte perceptivo intotalizable justamente porque está impregnado de lo invisible, es decir de una trascendencia que funge de condición de posibilidad de la propia visibilidad. Dicho de otro modo, lo invisible no es lo opuesto metafísico de lo visible sino su revés ontológico, así como el forro interno de un guante es el revés de su parte exterior, de la que resulta inseparable: «lo invisible no es lo contradictorio de lo visible: lo visible tiene una armazón de invisible, y lo in-visible es la contrapartida secreta de lo visible, sólo aparece en él, es el Nichturpräsentierbar [no-presentable de modo originario] que me es presentado como tal en el mundo - no se lo puede ver allí y todo esfuerzo para verlo allí lo hace desaparecer, pero está en la línea de lo visible, ese es su hogar virtual, se inscribe en él (entrelineas)»». ${ }^{22}$ Desde esta perspectiva, el sentido de ser del mundo concebido a partir del entrelazamiento ontológico de visible e invisible se fundaría en la presentación originaria de aquello que no es presentable de modo originario. ${ }^{23}$ Es decir, sobre la base de una negatividad o trascendencia originaria que es condición de posibilidad tanto de la percepción corpórea como del lenguaje pensante.

En este contexto teórico, el modo de ser del cuerpo subjetivo acaba siendo concebido también como un "pliegue" del Ser en el que éste se da un cuerpo visible y se hace pensamiento invisible simultáneamente. El propio Merleau-Ponty lo señala en otra nota de trabajo: «Querría desarrollar esto en el sentido: lo invisible es un hueco en lo visible, un pliegue (cursiva mía) en la pasividad, no producción pura. Para eso, hacer el análisis del lenguaje, que muestre hasta qué punto este es desplazamiento casi natural. [...] No hay que buscar cosas espirituales, sólo hay estructuras del vacío - Simplemente, quiero plantar ese vacío en el Ser visible, mostrar que es su reverso - en especial el reverso del lenguaje». ${ }^{24}$ El sujeto en cuanto pliegue se convierte, de este modo, en el lugar del tener lugar en el que la apertura originaria del Ser deja ver al mismo tiempo que hace hablar y pensar, ya que es el propio Ser quién se ve en lo visible y se piensa en lo invisible. En el marco de esta co-pertenencia ontológica de visible e invisible, la visión silenciosa de la percepción se descubre entrelazada al lenguaje pensante como expresión ideal y sublimación de la expresión bruta originaria procedente del cuerpo del mundo. Y esto porque el Ser del mundo lleva inscrito en sí mismo la posibilidad del lenguaje y del pensamiento como sublimación en el cuerpo del sujeto de las indefinidas posibilidades simbólicas latentes en él. En conclusión, lo que se puede sacar de todo este análisis es que esta concepción del Ser como articulación "quiasmática" de

21 Cfr. Ibid., p. 203 (enero de 1960).

22 Ibid., p. 191 (noviembre de 1959, trad. modificada).

23 Cfr. Ibid., p. 224 (mayo de 1960).

24 Ibid., p. 208 (febrero de 1960; trad. modificada). 
cuerpo y pensamiento que atraviesa al sujeto, y por el que éste está originariamente poseído, inaugura una irrevocable deconstrucción de la subjetividad. En cuanto "pliegue" de interior y exterior, de invisible y visible, la subjetividad se revela a sí misma constitutivamente supeditada a un movimiento inacabado y siempre por rehacer que no es propio del hombre sino del Ser en cuanto tal. ${ }^{25}$

A causa de este exceso del Ser sobre la conciencia del ser, la subjetividad se descubre vinculada a una trascendencia que es simultáneamente inmanente al ser del mundo y a sí misma. Insertada en un campo de operaciones procedentes del revés de las cosas, de la sombra de una latencia intotalizable que no cesa de alimentarla, la conciencia se descubre destronada de su posición de primacía metafísica. La subjetividad en cuanto "pliegue" del Ser ya no es definible como apoderamiento intelectual del mundo por parte de una interioridad constituyente; más bien, se revela destinada a una interrogación arqueológica del suelo ontológico del cual procede. Y la filosofía que realiza este esfuerzo arqueológico debe ser capaz de remontar a lo originario en la carne del mundo sin caer por ello en la ilusión de una fusión o coincidencia con el "origen". ${ }^{26}$

Como he intentado mostrar, en la filosofía del último Merleau-Ponty el cuerpo se manifiesta, de modo esencial, como "pliegue" de un Ser que ejerce de suelo ontológico para la dehiscencia tanto de la carne del cuerpo subjetivo como de la carne de todas las cosas. Según la nueva ontología esbozada en Le visible et l'invisible, el cuerpo ya no se puede pensar, como todavía parecía suceder en la Phénoménologie de la perception, a partir de la rehabilitación de su estatuto "trascendental". Considerado a partir de esta generatividad ontológica, el pensamiento que emerge del cuerpo hablante del sujeto se revela como un deseo de expresión que excede los límites del cogito tácito para hallar finalmente sus raíces en el principio de productividad inmanente a la trascendencia propia del Ser en cuanto carne del mundo.

\section{Bibliografía}

Barbaras, Renaud (1998): De la phénoménologie du corps à l'ontologie de la chair, en Id., Le tournant de l'experience. Recherches sur la philosophie de Merleau-Ponty, Paris, Vrin, 1998, pp. 95-136.

Barbaras, Renaud (2003): «Le problème du chiasme», en Studia Phanomenologica, Vol. III, no 3-4, pp. 15-20.

Dastur, Françoise (2001): Le corps de la parole, en Id., Chair et langage, La Versanne, Encre Marine, pp. 49-68.

Deleuze, Gilles (1986): Foucault, Paris, Minuit.

$25 \mathrm{Al}$ respecto, véase este breve pero no por ello menos esclarecedor apunte de Deleuze: «Un Dehors, plus lointain que tout extérieur, "se tord", "se plie", "se double" d'un Dedans, plus profond que tout intérieur, et rend seul possible le rapport derivé de l'intérieur avec l'extérieur. C'est même cette torsion qui définit la "Chair", au delà du corps propre et de ses objets. Bref, l'intentionalité de l'étant se dépasse vers le pli de l'être, vers l'Être comme pli». Cfr. Deleuze, G., Foucault, Paris, Minuit, 1986, p. 117. Véase también: Gambazzi, P., «La piega e il pensiero. Sull'ontologia di Merleau-Ponty», Aut-Aut, no 262-263, 1994, pp. 21-47.

26 Sobre el concepto de arqueología véase : Lawlor, L. «Le chiasme et le pli. Une introduction au concept philosophique d'archéologie», en Cariou, M., Barbaras, R., Bimbenet, E. (eds), Merleau-Ponty aux frontières de l'invisible, Cahiers de Chiasmi International, $\mathrm{n}^{\circ}$ 1, 2003, pp. 191-205. 
Descartes, René (1977): Meditaciones metafísicas, Madrid, Alfaguara.

Firenze, Antonino (2007): «I segni di una nuova concezione dell'essere. Merleau-Ponty lettore di Heidegger», Chiasmi international, no 9, pp. 169-190.

Firenze, Antonino (2010): «Mundo-de-la-vida, intercorporeidad, Tierra: Merleau-Ponty y la fenomenología», Phainomenon, ${ }^{\circ}$ 20, pp. 129-142.

Firenze, Antonino (2011): Il corpo e l'impensato. Saggio su Merleau-Ponty, Milano, Mimesis.

Gambazzi, Paolo (1994): «La piega e il pensiero. Sull'ontologia di Merleau-Ponty», Aut-Aut, $n^{\circ} 262-263$, pp. 21-47.

Husserl, Edmund (1940): «Umsturz der Köpernikanischen Lehre in der gewöhnlichen weltanschaulichen Interpretation», en Farber, M. (ed.), Philosophical Essays in Memory of E. Husserl, Cambridge, Harvard University Press, pp. 307-325.

Lawlor, Leonard (2003): «Le chiasme et le pli. Une introduction au concept philosophique d'archéologie», en Cariou, M., Barbaras, R., Bimbenet, E. (eds), Merleau-Ponty aux frontières de l'invisible, Cahiers de Chiasmi International, $\mathrm{n}^{\circ} 1$, Milano, Mimesis, pp. 191-205.

Merleau-Ponty, Maurice (2002): La structure du comportement, Paris, Puf; trad. cast. de E. Alonso, La estructura del comportamiento, Buenos Aires, Hachette, 1957.

Merleau-Ponty, Maurice (2003): Phénoménologie de la perception, Paris, Gallimard; trad. cast. de J. Cabanes, Fenomenología de la percepción, México D.F. - Buenos Aires, Planeta De Agostini, 1993.

Merleau-Ponty, Maurice (2003): Le visible et l'invisible, seguido de: Notas de trabajo, texto establecido por C. Lefort, Gallimard, Paris; trad. cast. de E. Consigli, B. Capdevielle, Lo visible y lo invisible, Buenos Aires, Nueva Visión, 2010.

Nancy, Jean-Luc (2000): Corpus, Paris, Éditions Métailié.

Saint Aubert, Emanuel DE (2006), Vers une ontologie indirecte. Sources et enjeux critiques de l'appel à l'ontologie chez Merleau-Ponty, Paris, Vrin.

Taminiaux, Jacques, «L'experience, l'expression et la forme dans l'itineraire de MerleauPonty», en Id., Le regard et l'excédent, La Haye, Martinus Nijhoff, 1977, pp. 90-115.

Thierry, Yves (1987): Du corps parlant. Le langage chez Merleau-Ponty, Bruxelles, Ousia.

Thierry, Yves (1998): «Le 'cogito' comme expérience sensible» en Barbaras, R., Robert, F. (ed.), Notes de Cours sur L'origine de la géométrie de Husserl. Suivi de «Recherche sur la phénoménologie de Merleau-Ponty», Paris, PUF, pp. 255-268. 